\title{
THE ASYMPTOTIC RESPONSE OF A CALORIMETER
}

\author{
A. McNABB
}

(Received 8 April 1980)

\begin{abstract}
An algorithm is given for calculating the asymptotic behaviour of the temperature of the fluid in an adiabatic calorimeter, and used to derive the asymptote for a finite cylinder.
\end{abstract}

\section{Introduction}

This note is concerned with the temperature changes which occur in the fluid of a calorimeter when a body at a different temperature is immersed in it. Even for bodies of a simple shape, and assuming these to be homogeneous isotropic conductors, with the fluid in the calorimeter well stirred and no heat loss from the system, it is not always easy to calculate the theoretical behaviour. However, we show that the asymptotic behaviour of the fluid temperature is more easily obtained, and present formulae describing this for the case of a finite cylinder by way of illustration.

Imagine a homogeneous isotropic conductor, at a uniform temperature $T_{0}$, quickly immersed in the well-stirred fluid of a thermally insulated calorimeter which was initially at a uniform temperature $T_{1}$. The temperature response of the conductor is assumed to be governed by the diffusion equation

$$
K \nabla^{2} T=\rho C \frac{\partial T}{\partial t} \text { in } \mathcal{G},
$$

where $\mathcal{G}$ is the region occupied by the conducting body, and by the boundary and initial conditions

$$
T=T_{0} \text { in } \mathcal{G} \text { at } t=0 \text { and } T=\mathcal{T}(t) \text { on } \partial \mathcal{G},
$$

where $\mathcal{T}(t)$ is the calorimeter temperature at time $t$ and $\partial \mathcal{G}$ is the bounding surface of $\mathcal{G}$. 
The system is imagined to be completely insulated thermally so that, at all cimes $t \geqslant 0$, the total energy content of the calorimeter, fluid and conductor is constant. Let $V_{f}, \rho_{f}, C_{f} ; V_{c}, \rho_{c}, C_{c} ; V, \rho, C$ denote the volumes, densities and specific heats of the fluid, calorimeter and conducting body respectively. Then this fixed total energy requirement ensures that

$$
\begin{aligned}
\left(V_{f} \rho_{f} C_{f}+V_{c} \rho_{c} C_{c}\right) \sigma(t) & +\rho C \iint_{\mathcal{G}} \int T d \tau=\left(V_{f} \rho_{f} C_{f}+V_{c} \rho_{c} C_{c}\right) T_{1}+V \rho C T_{0} \\
& =\left(V_{f} \rho_{f} C_{f}+V_{c} \rho_{c} C_{c}+V \rho C\right) T_{\infty},(2)
\end{aligned}
$$

where $T_{\infty}$ is the final equilibrium temperature of the system. Equations (2) can be used to calculate $T_{\infty}$.

Suppose for the sake of being definite that $T_{1}>T_{0}$, and we plot $\sigma(t)$ against $t$. Then $T$ starts at $T_{1}$ and decreases towards $T_{\infty}$, ultimately in a simple exponential fashion. The integral $\phi(t)$ defined by

$$
\phi(t) \equiv \int_{0}^{t} \frac{\sigma(t)-T_{\infty}}{T_{1}-T_{\infty}} d t
$$

tends to a limit $\phi_{\infty}$ as $t$ tends to infinity, and we show below how $\phi_{\infty}$ may be calculated.

\section{Calculation of $\phi_{\infty}$}

Define the function

$$
\theta(\mathbf{x})=\int_{0}^{\infty} \frac{T(\mathbf{x}, t)-T_{\infty}}{T_{0}-T_{\infty}} d t \quad \text { for } \mathbf{x} \in \mathcal{G},
$$

and note that equation (1) implies

$$
\left(T_{0}-T_{\infty}\right) \nabla^{2} \theta=\frac{\rho C}{K} \int_{0}^{\infty} \frac{\partial T}{\partial t} d t=\frac{\rho C}{K}\left(T_{\infty}-T_{0}\right)
$$

so that

$$
\nabla^{2} \theta=-\frac{\rho C}{K} \quad \text { for } \mathbf{x} \in \mathcal{G} .
$$

From equation (2), we also have

$$
\left(V_{f} \rho_{f} C_{f}+V_{c} \rho_{c} C_{c}\right)\left(\tau(t)-T_{\infty}\right)+\rho C \iint_{\mathcal{G}} \int\left(T-T_{\infty}\right) d \tau=0,
$$

and so

$$
\begin{aligned}
\iint_{\mathcal{G}} \int \theta(\mathbf{x}) d \tau & =\int_{0}^{\infty} \iint_{\mathcal{G}} \int \frac{T-T_{\infty}}{T_{0}-T_{\infty}} d \tau d t \\
& =V \int_{0}^{\infty} \frac{\mathcal{T}-T_{\infty}}{T_{1}-T_{\infty}} d t=V \phi_{\infty} .
\end{aligned}
$$


Now, on the boundary $\partial \mathcal{G}$ of $\mathcal{G}$, we have $T=\mathcal{T}$ for all $t>0$, so that

$$
\int_{0}^{\infty}\left(T(\mathbf{x}, t)-T_{\infty}\right) d t=\int_{0}^{\infty}\left(\mathcal{T}-T_{\infty}\right) d t \text { for } \mathbf{x} \in \partial \mathcal{G},
$$

or

$$
\theta(\mathbf{x})=-\frac{T_{1}-T_{\infty}}{T_{\infty}-T_{0}} \phi_{\infty} \quad \text { for } \mathbf{x} \in \partial \mathcal{G} .
$$

Suppose $u$ satisfies the Poisson equation,

$$
\nabla^{2} u=-1 \quad \text { in } \mathcal{G} \text {, with } u=0 \text { on } \partial \mathcal{G} .
$$

Then

$$
\theta(\mathbf{x})=\frac{\rho C}{K} u(\mathbf{x})-\frac{T_{1}-T_{\infty}}{T_{\infty}-T_{0}} \phi_{\infty} \quad \text { for } \mathbf{x} \in \mathcal{G},
$$

and from equation (7) we see $\phi_{\infty}$ satisfies the equation

$$
\frac{\rho C}{K} \iiint_{\mathcal{G}} u d \tau-\frac{T_{1}-T_{\infty}}{T_{\infty}-T_{0}} V \phi_{\infty}=V \phi_{\infty},
$$

so that

$$
\phi_{\infty}=\frac{T_{\infty}-T_{0}}{T_{1}-T_{0}} \frac{\rho C}{K V} \iiint_{\mathcal{G}} u d \tau .
$$

Evidently, for a conductor of given shape defining a region $\mathcal{G}$, we need to calculate the mean value,

$$
I=\frac{1}{V} \iint_{\mathcal{G}} u d \tau
$$

where $u$ satisfies the system (9).

\section{A general formula for $I$}

Let $G\left(\mathbf{x} \mid \mathrm{x}_{0}\right)$ be the Green's function satisfying

$$
\nabla^{2} G=-\delta\left(\mathbf{x}-\mathbf{x}_{0}\right) \quad \text { for } \mathbf{x}, \mathbf{x}_{0} \in \mathcal{G},
$$

where $\delta$ is the Dirac Delta function.

Then $G$ can be written in the form

$$
G\left(\mathbf{x} \mid \mathbf{x}_{0}\right)=\sum_{n} \frac{\bar{\phi}_{n}\left(\mathbf{x}_{0}\right) \phi_{n}(\mathbf{x})}{k_{n}^{2}} \text { for } \mathbf{x}_{0}, \mathbf{x} \in \mathcal{G},
$$

where $\phi_{n}$ are a complete set of eigenfunctions with eigenvalues $k_{n}^{2}$ of the equations

$$
\nabla^{2} \phi_{n}+k_{n}^{2} \phi_{n}=0 \text { in } \mathcal{G}, \quad \phi_{n}=0 \text { on } \partial \mathcal{G}
$$


rormalized so that

$$
\iint_{\mathcal{G}} \int \bar{\phi}_{n} \phi_{m} d \tau=\delta_{n, m}
$$

(see Morse and Feshbach [1], Section 7.2). Then

$$
u(\mathbf{x})=\iint_{\mathcal{S}_{0}} \int G\left(\mathbf{x} \mid \mathbf{x}_{0}\right) d \tau_{0}
$$

and

$$
\begin{aligned}
I & =\frac{1}{V} \iint_{\mathcal{G}} \int u(\mathbf{x}) d \tau=\frac{1}{V} \iiint_{\mathcal{G}} \iiint_{\mathcal{G}_{0}} \int_{G} G\left(\mathbf{x} \mid \mathbf{x}_{0}\right) d \tau_{0} d \tau \\
& =\frac{1}{V} \sum_{n}\left|\iint_{\mathcal{G}} \int \frac{\phi_{n}(\mathbf{x}) d \tau}{k_{n}}\right|^{2} .
\end{aligned}
$$

\section{An example}

Consider the case where $\mathcal{G}$ is a cylinder of radius $a$ and height $2 h$. For this example we find, using cylindrical polar coordinates $(r, z)$, that

$$
\begin{aligned}
V & =2 \pi a^{2} h, \\
\phi_{n} & =\frac{\sqrt{2}}{\sqrt{V} J_{1}\left(C_{l}\right)} J_{0}\left(C_{l} \frac{r}{a}\right) \cos \left(d_{m} \frac{z}{h}\right), \\
k_{n}^{2} & =\frac{C_{l}^{2}}{a^{2}}+\frac{d_{m}^{2}}{h^{2}} \equiv k_{l, m},
\end{aligned}
$$

where $C_{l}, d_{m}$ are the positive zeros of $J_{0}(z)$ and $\cos (z)$, respectively (that is, $\left.d_{m}=(2 m-1) \pi / 2, m>0\right)$. Thus

$$
\iiint_{\mathcal{G}} \phi_{n} d \tau=(-1)^{m+1} \frac{8 \sqrt{2} a^{2} h}{(2 m-1) C_{l} \sqrt{V}},
$$

and so

$$
I=8 \sum_{l, m>0} \frac{1}{d_{m}^{2} C_{l}^{2} k_{l, m}^{2}} .
$$

We may use the method of contour integration to evaluate partial sums over either $l$ or $m$ (see Phillips [2], Section 50, for a description of this method). Thus we find, on summing over $l$, that

$$
I=2 h^{2}\left\{\frac{1}{6}-\sum_{m=1}^{\infty} \frac{I_{1}\left(z_{m}\right)}{b_{m}^{4} z_{m} I_{0}\left(z_{m}\right)}\right\},
$$


where $b_{m}=\left(m-\frac{1}{2}\right) \pi$ and $z_{m}=a b_{m} / h$, and, on summing over $m$, that

$$
I=2 a^{2}\left\{\frac{1}{16}-2 \sum_{l=1}^{\infty} \frac{\tanh \left(z_{l}\right)}{z_{l} C_{l}^{4}}\right\},
$$

where $z_{l}=C_{l} h / a$. Formula (17) is particularly appropriate when $a \gg h$ and (18) is more useful in the reverse situation when $h \gg a$.

\section{Practical applications to calorimetry}

Our function $\phi_{\infty}$ can be written in the form

$$
\phi_{\infty} \equiv \int_{0}^{\infty} \frac{\sigma-T_{\infty}}{T_{1}-T_{\infty}} d t=-\int_{0}^{\infty} \frac{t \frac{\partial \mathcal{T}}{\partial t}}{T_{1}-T_{\infty}} d t
$$

and so the expression $\phi_{\infty}$ can be regarded as a measure of the time the action occurs. Equations (11), (12) and (19) give the formula

$$
\int_{0}^{\infty} \frac{\sigma-T_{\infty}}{T_{1}-T_{\infty}} d t=\frac{T_{\infty}-T_{0}}{T_{1}-T_{0}} \frac{\rho C}{K} I,
$$

where $I$ is the geometric factor defined by equation (12). If the integral on the left is computed from the experimental results for $\mathcal{T}$, then equation (20) may be used to compute $K / \rho C$, the thermal diffusivity of the material of the immersed conductor. Since equation (2) gives the specific heat $\rho C$, the thermal conductivity may be determined.

\section{References}

[1] Philip M. Morse and Herman Feshbach, Methods of theoretical physics, (McGraw-Hill, 1953).

[2] E. G. Phillips, Functions of a complex variable with applications, (Oliver and Boyd, 1949).

Applied Mathematics Division

Department of Scientific and Industrial Research

Wellington

New Zealand 